\title{
Calculation of Thermal Neutron Induced Electron Fluxes Generated in a Fiber-Optic Cerenkov Radiation Sensor Using Monte Carlo N-Particle Transport Code
}

\author{
Kyoung Won Jang, Sang Hun Shin, Wook Jae Yoo, \\ Seunghyun Cho, ${ }^{1}$ and Bongsoo Lee ${ }^{2 *}$ \\ School of Biomedical Engineering, BK21 Plus Research Institute of Biomedical Engineering, Konkuk University, \\ 268 Chungwon-daero, Chungju-si, Chungcheongbuk-do, 27478, Korea \\ ${ }^{1}$ Department of Organic Materials \& Fiber Engineering, College of Engineering, Soongsil University, \\ 369 Sangdo-ro, Dongjak-gu, Seoul-si, 06978, Korea \\ ${ }^{2}$ School of Energy Systems Engineering, College of Engineering, Chung-Ang University, \\ 84 Heukseok-ro, Dongjak-gu, Seoul-si, 06974, Korea
}

(Received March 2, 2015; accepted April 22, 2016)

Keywords: fiber-optic radiation sensor, MCNPX simulation, Cerenkov radiation, thermal neutron, electron flux

The purpose of this research is to develop a fiber-optic Cerenkov radiation sensor (FOCRS) for detecting thermal neutrons in mixed radiation fields. Thus, we designed a probe for FOCRS to detect thermal neutrons and background gamma rays using a Gd-157 foil and rutile crystal. A probe for a reference sensor to detect gamma rays was also designed with a rutile crystal. To clarify the relationship between electron fluxes and intensities of Cerenkov radiation, the electron fluxes inducing Cerenkov radiation in the probes of FOCRS and the reference sensor were calculated using Monte Carlo N-particle transport code (MCNPX) simulations.

\section{Introduction}

Generally, fiber-optic radiation sensors (FORSs) for detecting neutrons consist of neutron converters, optical fibers, and scintillators. ${ }^{(1,2)}$ These types of sensors are immune to environmental influences such as pressure, humidity, and electromagnetic field. ${ }^{(3-5)}$ In addition, their small radii and thinness make it possible to detect neutrons with high spatial resolution in a narrow space. Moreover, specific optical fibers, such as metal coated optical fibers, allow the FORS to be used under conditions of high temperature. However, although conventional scintillators have high scintillation efficiencies and short decay times, these types of materials have some disadvantages under specific conditions. In high ionization density, scintillation molecules can be temporarily damaged by high energy charged particles. This phenomenon, which is known as ionization quenching, causes non-proportionality between energy losses of charged particles and scintillation outputs. $^{(6)}$ In addition, the light yields of scintillators vary with ambient temperature, and this characteristic restricts the use of a scintillator at high temperatures. Therefore, a novel technique is required to detect neutrons in extremely harsh environments such as in the molten-salts in pyroprocessing or the cores of nuclear reactors.

*Corresponding author: e-mail: bslee@cau.ac.kr 
A charged particle cannot travel with a velocity greater than the phase velocity of light in a vacuum. However, in some dielectric media, including water, silica, and polymethyl methacrylate (PMMA), a high energy particle can pass through the media with a velocity greater than the phase velocity of light. When the charged particle does this, the electromagnetic field close to the particle polarizes the medium along its path, which causes the electrons in the atoms to follow the waveform of the pulse. In contrast to the scintillation generated in a scintillator, Cerenkov radiation generated from a radiator can be used under extremely harsh conditions. In a high temperature tokamak, electron fluxes can be obtained by measuring the Cerenkov radiation generated from some crystals. (7) Also, in radiotherapy dosimetry, it is possible to measure the relative depth of doses for proton beams without ionization quenching by measuring the intensity of Cerenkov radiation. ${ }^{(8)}$ Therefore, Cerenkov radiation generated in radiators can be a significant signal under conditions of hazardous radiation.

To detect neutrons using the Cerenkov effect, we have fabricated a fiber-optic Cerenkov radiation sensor (FOCRS) using a Gd-foil, a rutile crystal, and an optical fiber, and have measured pure thermal neutrons successfully.(9) However, to apply the FOCRS in actual nuclear facilities, the FOCRS should detect the thermal neutrons selectively in mixed radiation fields. The purpose of this research is to develop FOCRS for detecting thermal neutrons in mixed radiation fields. In this study, we designed a probe for FOCRS to detect neutrons and gamma rays using a Gd-157 foil and a rutile crystal. The probe of a reference sensor for detecting gamma rays was also designed with the rutile crystal. To clarify the relationship between electron fluxes and intensities of Cerenkov radiation, the electron fluxes inducing Cerenkov radiation in the probes of FOCRS and the reference sensor were calculated using Monte Carlo N-particle transport code (MCNPX) simulations.

\section{Materials and Methods}

In our simulations, a Gd-157 foil is used as a neutron converter. The Gd-157 foil has a cross section for a thermal neutron of 255000 barns, which is among the highest nuclear cross sections found in any material. After interaction with the neutrons, the Gd-157 emits gamma rays with energies up to $7.8 \mathrm{MeV}$ and $72 \mathrm{keV}$ conversion electrons. ${ }^{(10)}$ The gamma rays and electrons emitted from the Gd-157 induced by the neutrons have sufficient energy to produce Cerenkov radiation in a dielectric medium having a high refractive index. The dimensions and density of the Gd- 157 foil used in this research are $5 \times 5 \times 0.025 \mathrm{~mm}^{3}$ and $7.9 \mathrm{~g} / \mathrm{cm}^{3}$.

As a Cerenkov radiator, rutile crystals $\left(\mathrm{TiO}_{2}\right)$ were employed throughout this research. In general, Cerenkov radiators should have high refractive indices to produce Cerenkov radiation for low energy electrons. The rutile crystals have a refractive index of 2.87 at a wavelength of $430 \mathrm{~nm}$. The Cerenkov threshold energy (CTE) of electrons in the crystals required to produce Cerenkov radiation was calculated as $34 \mathrm{keV}$ using Eq. (1);

$$
E_{\mathrm{Th}}=m_{0} c^{2}\left(\frac{n}{\sqrt{n^{2}-1}}-1\right),
$$

where $m_{0}$ is the rest mass of the charged particle, $c$ is the phase velocity of light, and $n$ is the refractive index of the Cerenkov radiator. Since this energy is smaller than that of the conversion electrons and gamma rays emitted from the Gd-157, Cerenkov radiation can be produced in the rutile crystals. The dimensions and density of rutile crystals used in this research are $10 \times 10 \times 0.1$ $\mathrm{mm}^{3}$ and $4.23 \mathrm{~g} / \mathrm{cm}^{3}$. 
Figure 1 shows the MCNPX simulation scheme. In our simulations, a Cf-252 source which emits neutrons with a mean energy of about $2 \mathrm{MeV}$ was used as the neutron source. A polyethylene (PE) block with a thickness from 5 to $45 \mathrm{~mm}$ was placed to increase the number of thermal neutrons between the source and the sensor probes. In our simulations, a sensor probe consisting of the rutile crystal and the Gd-157 foil was used to detect thermal neutrons and gamma rays, and a sensor probe consisting of only the rutile crystal was used for gamma ray detection. The fluxes of neutrons and electrons in the sensor probes were calculated using tally4.

\section{Results and Discussion}

Figure 2 shows calculated energy distributions of electrons in the rutile crystals. These results were obtained for the 4-cm thick PE using the MCNPX simulation. In the case of the rutile crystal with a Gd-157 foil, the peak electron flux was in an energy range below $34 \mathrm{keV}$. As mentioned previously, because the CTE of electrons in the crystal is about $34 \mathrm{keV}$, the electrons in this range cannot contribute to producing Cerenkov radiation in the rutile crystal. Most of these electrons are derived from conversion electrons with an energy of $72 \mathrm{keV}$ emitted from the Gd-157 foil and background gamma rays; the conversion electrons can lose their energy to a level below the CTE by passing through the Gd-157 foil and rutile crystal.

On the other hand, gamma rays generated from the Gd-157 have relatively high energies of up to $7.8 \mathrm{MeV}$; thus, subsequent electrons induced by interactions between the gamma rays and the rutile crystals have sufficient energy to produce Cerenkov radiation in the crystals. Therefore, we can estimate that almost all Cerenkov radiation generated in the FOCRS is induced by the gamma rays generated from the Gd-157. The result from the rutile crystal without a Gd-157 foil presents the electron fluxes induced by background gamma rays. Typically, background gamma rays are produced by interactions between neutrons and PE. In our simulation, the total electron flux of the rutile crystal with Gd-157 foil was 4.2 times higher than that of the rutile crystal without the Gd157.

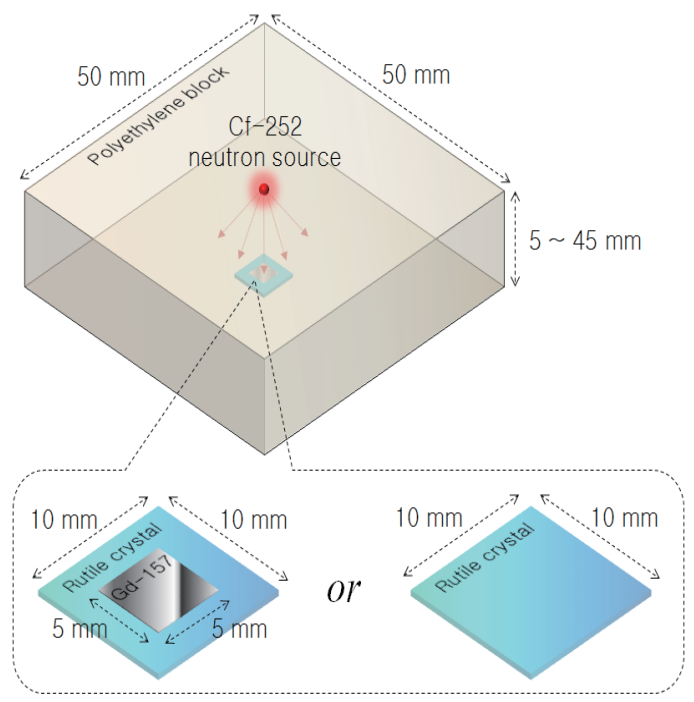

Fig. 1. (Color online) MCNPX simulation scheme.

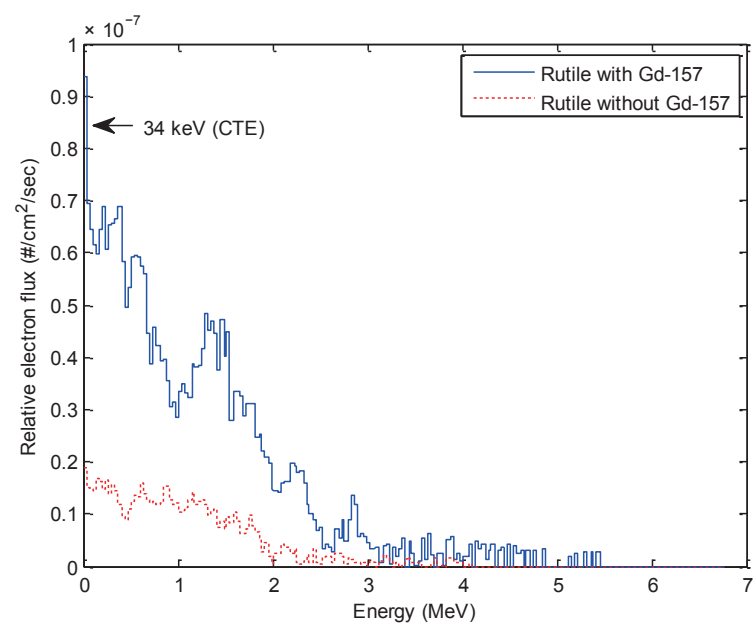

Fig. 2. (Color online) Calculated energy distributions of electrons in rutile crystals. 


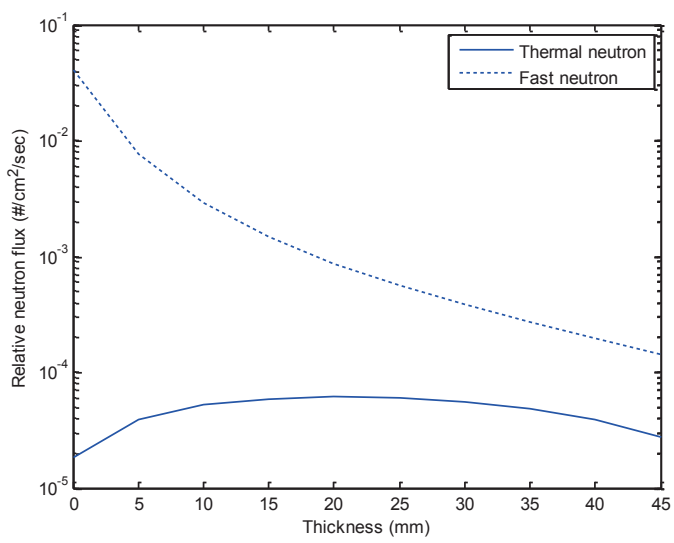

(a)

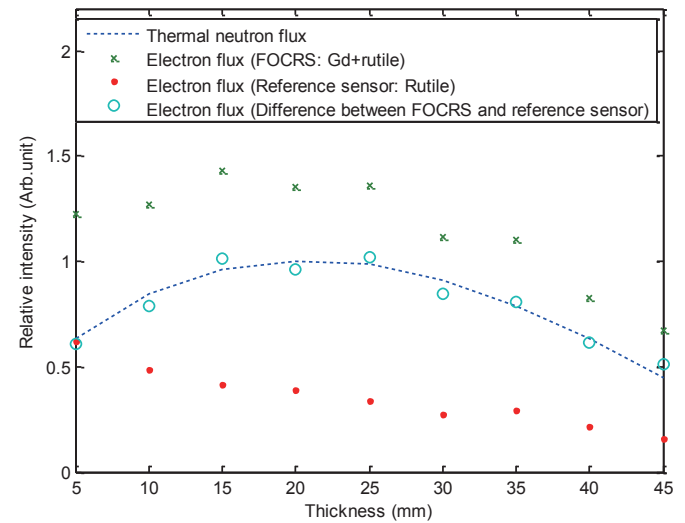

(b)

Fig. 3. (Color online) Calculated fluxes of (a) neutrons and (b) electrons in rutile crystals as a function of PE thicknesses.

The calculated neutron fluxes in PE caused by Cf-252 are shown in Fig. 3(a). In general, most neutrons emitted from the Cf-252 isotope are fast neutrons having energies over $1 \mathrm{MeV}$. In our simulations, therefore, the fluxes of fast neutrons account for greater portions of total neutron fluxes in PE. Because the fast neutrons lose their energy by passing through PE, their fluxes decreased according to the thickness of the PE. Meanwhile, most thermal neutrons are derived from fast neutrons. Therefore, the fluxes of thermal neutrons increased for PE thickness up to $20 \mathrm{~mm}$ and then decreased as PE thickness was increased beyond $20 \mathrm{~mm}$.

Figure 3(b) shows calculated electron fluxes over CTE in rutile crystals with and without Gd157 foil for various PE thicknesses. Since the ultimate purpose of this study was to detect thermal neutrons by measuring intensities of Cerenkov radiation induced by electrons and gamma rays emitted from Gd-157, the intensities caused by the background gamma rays had to be eliminated. Therefore, we subtracted the electron fluxes of the reference sensor from that of the FOCRS. The differences in electron fluxes between the FOCRS and the reference sensor are in good agreement with the thermal neutron fluxes. The difference between the calculated fluxes of electrons and thermal neutrons was about $4.3 \%$.

\section{Conclusions}

By measuring the intensity of Cerenkov radiation generated in a dielectric medium, it is possible to detect some radiations without using scintillation material. In this research, we proposed a novel method for detecting thermal neutrons in mixed radiation fields with a FOCRS. The probe of FOCRS for detecting thermal neutrons was designed using a Gd-157 foil and a rutile crystal. The probe of the reference sensor was also designed with a rutile crystal to detect background gamma rays. To clarify the relationship between electron flux and intensity of Cerenkov radiation, electron fluxes inducing Cerenkov radiation in the FOCRS and the reference sensor were calculated using the MCNPX code. Finally, relative fluxes of thermal neutrons in a PE generated by a Cf- 252 neutron source were compared with the calculated electron fluxes of the FOCRS and the reference sensor. As a result, the differences in electron flux between the FOCRS and the reference sensor were very close to calculated thermal neutron fluxes. 
Further studies will be carried out to fabricate FOCRS for detecting neutrons at high temperatures. It is anticipated that the novel and simple FOCRS using the Cerenkov effect proposed here can be effectively used to measure radiation in nuclear facilities.

\section{Acknowledgements}

This research was supported by the Basic Science Research Program through the National Research Foundation of Korea (NRF) funded by the Ministry of Science, ICT \& Future Planning and this work was supported by the Nuclear Safety Research Program through the Korea Foundation Of Nuclear Safety (KOFONS), granted financial resource from the Nuclear Safety and Security Commission (NSSC), Republic of Korea (Nos. 2013R1A1A1061647, 2014R1A2A2A04002620, and 1403017-0114-SB110). Also, this research was supported by the National Nuclear R\&D Program through the National Research Foundation of Korea (NRF) funded by the Ministry of Science, ICT and future Planning (No. 2015M2B2A4031190).

\section{References}

1 T. K. McKnight, J. B. Czirr, K. Littrell, and B. J. Campbell: Nucl. Instrum. Meth. A 586 (2008) 246.

2 M. Ishikawa, K. Ono, Y. Sakurai, H. Unesaki, A. Uritani, G. Bengua, T. Kobayashi, K. Tanaka, and T. Kosako: Appl. Radiat. Isotopes 61 (2004) 775.

3 A. F. Fernandez, B. Brichard, S. O'Keeffe, C. Fitzpatrick, E. Lewis, J.-R. Vaille, L. Dusseau, D. A. Jackson, F. Ravotti, M. Glaser, and H. El-Rabii: Fusion Eng. Des. 83 (2008) 50.

4 S. O'Keeffe, C. Fitzpatrick, E. Lewis, and A. I. Al-shamma'a: Sensor Rev. 28 (2008) 136.

5 B. Lee, W. Y. Choi, and J. K. Walker: Polym. Eng. Sci. 40 (2000) 1996.

6 S. Mouatassim, G. J. Costa, G. Guillaume, B. Heusch, A. Huck, and M. Moszynski: Nucl. Instrum. Meth. A 359 (1995) 530.

7 L. Jakubowski, M. J. Sadowski, J. Zebrowski, M. Rabinski, K. Malinowski, R. Mirowski, Ph. Lotte, J. Gunn, J.-Y. Pascal, G. Colledani, V. Basiuk, M. Goniche, and M. Lipa: Rev. Sci. Instrum. 81 (2010) 013504.

8 K. W. Jang, W. J. Yoo, S. H. Shin, D. Shin, and B. Lee: Opt. Express 20 (2012) 13907.

9 K. W. Jang, T. Yagi, C. H. Pyeon, W. J. Yoo, S. H. Shin, T. Misawa, and B. Lee: Opt. Express 21 (2013) 14573.

10 G. D. Stasio, P. Casalbore, R. Pallini, B. Gilbert, F. Sanita, M. T. Ciotti, G. Rosi, A. Festinesi, L. M. Larocca, A. Rinelli, D. Perret, D. W. Mogk, P. Perfetti, M. P. Mehta, and D. Mercanti: Cancer Res. 61 (2001) 4272. 